\title{
Evolution of Genetic Organization in Digital Organisms
}

\author{
Charles Ofria and Christoph Adami \\ Beckman Institute and Kellogg Radiation Laboratory \\ California Institute of Technology \\ Pasadena, CA 91125
}

\begin{abstract}
We examine the evolution of expression patterns and the organization of genetic information in populations of self-replicating digital organisms. Seeding the experiments with a linearly expressed ancestor, we witness the development of complex, parallel secondary expression patterns. Using principles from information theory, we demonstrate an evolutionary pressure towards overlapping expressions causing variation (and hence further evolution) to sharply drop. Finally, we compare the overlapping sections of dominant genomes to those portions which are singly expressed and observe a significant difference in the entropy of their encoding.
\end{abstract}

\section{Introduction}

Life on Earth is the product of approximately four billion years of evolution, with the vast majority of beginning and intermediate states lost to us forever. The exact details of how we evolved to become what we are may be impossible to ascertain for sure, but we may still be able to better understand the evolutionary pressures exerted on life, and from that reconstruct sections of the path our evolution is likely to have taken.

Here we look at a fundamental issue to life as we know it; the organization of the genetic code and the differentiation in its expression. DNA is structured into many distinct genes which can be concurrently active, transcribed and expressed in an asynchronous, (i.e., differentiated) manner. Extant living systems have evolved to a state where multiple genes influence each other, typically without sharing genetic material. It appears that in all higher life forms each gene has its own unique position on the genome, while the transcription products often interact with unique positions "downstream". Those organisms which do exhibit overlapping expression patterns are mostly virii and bacteriophages [9]. This suggests that genomes containing only purely localized, non-overlapping genes must have evolved later on [6].

Upon initial inspection, the reason for a spatially separated layout appears uncertain. A modular design may be quite common in artificially created coding schemes such as computer programs, but, in fact, only reflects a designer's quest to create human-understandable structures. Evolution has no such incentive, and will always exert pressure towards the most immediate solution given the current circumstances. A more compressed coding scheme, perhaps 
with overlapping genes, would allow a sufficiently shorter code that would minimize the mutational load and hence be able to preserve its information with a higher degree of accuracy. Furthermore, such overlapping regions might be used for gene regulation. Why this is not much more common becomes clearer when we observe those examples from nature where these overlapping reading frames do exist, such as DNA phages [9] and eukaryotic viruses [14]. Even in these organisms only some sections of code overlap, but examination of those sections reveals that they contain little variation - almost all of the nucleotides are effectively frozen in their current state from one generation to the next [7, [8]. This occurs because for any mutation to be neutral in such a section of genetic code, it must be neutral to both of the genes which it would affect. Further, most of the mutations that occur in DNA which are neutral occur in the third nucleotide of a codon, as substitutions in that position are often synonymous. When overlapping genes have offset (out-of-phase) reading frames, however, the position of the third nucleotide in one gene maps to the first or second in the other, leaving no redundancy.

We have investigated the development of genome organization and differentiation in digital organisms: populations of self-replicating computer-code living in a computer's memory. Such "Artificial Life" systems have proven to be useful test cases to investigate the biochemical paradigm because the computational chemistry the digital organisms are based on share Turing universality with their biochemical cousins, i.e., just as any type of organism appears to be implementable in biochemistry, the digital organisms can in principle compute any (partially-recursive) function [2]. Due to the ease with which experiments can be prepared, data can be gathered, and trials can be repeated, digital organisms present an important tool to study universal traits in the evolution and development of symbolic sequences. Differentiation in digital organisms was first investigated within the tierra architecture [12, 15, 13, and we comment on those results below.

For the present study, we have extended our avida system [2] to allow for the expression of a second gene to occur in parallel. We then processed the evolution of 600 populations from a seed program to complex informationprocessing sequences for an average of over 9000 generations each. The 600 trials were divided into four sets which differ in the length of the seed program, constraints on size evolution, and their ability to express multiple portions of code in parallel. All populations with a genetic basis allowing for the development of multiple threads learn to use them almost immediately (each thread is an instruction pointer which executes the code independently), but the methods by which this happens are quite distinct and varied. In the next section, we outline the most important design characteristics of the avida system, focusing mostly on the particular experimental setup needed for this study. Also, we outline the kind of observables which we record, and discuss measures of differentiation. In Section 3 we present results obtained with our multiple-expression digital chemistry and compare them to controls in which 
no secondary expression was allowed. In Section 4 we study the evolution of differentiation for different experimental boundary conditions, while Section 5 explores in more detail the organization and development of genes at the hand of an example. We close in Section 6 with a discussion of the evidence and conclusions, and issue caveats about applying the lessons learned directly to biochemistry.

\section{Experimental Details}

\subsection{The Avida Platform}

The computer program avida is an auto-adaptive genetic system [1] designed primarily for use as a platform in Artificial Life research. The system consists of a population of self-reproducing strings of instructions with a Turingcomplete genetic basis subjected to Poisson-random mutations during reproduction. The population adapts to a combination of an intrinsic fitness criterion (self-reproduction) and an externally imposed (extrinsic) fitness landscape provided by the researcher by creating an information-rich environment.

A normal avida organism is a computer program written in a very simple assembly language, with 28 possible commands for each line (Table I).

Table 1. Standard (single expression) avida instruction set

\begin{tabular}{|c|c|}
\hline Instruction type & Mnemonic \\
\hline flow control & jump-b, jump-f, call, return \\
conditionals & if-n-eq, if-less, if-bit-1 \\
self analysis & search-f, search-b \\
computation & shift-l, shift-r, inc, dec, swap \\
& swap-stk, push, pop, add, sub, nand \\
metabolic & alloc, divide, copy \\
I/O & get, put \\
labels & nop-A, nop-B, nop-C \\
\hline
\end{tabular}

These programs exist on a two-dimensional lattice with toroidal boundary conditions, and are executed on simple virtual CPUs residing at the lattice-sites which process their code allowing them to interact with their environment and perform functions such as self-replication, as well computations on numbers which are found in the external environment. For more details on the virtual CPUs in avida, see [10].

In order to study the evolution of code expression, we have extended the instruction set of Table I to allow for more than one instruction pointer to execute a program's code. Within the biochemical metaphor, the simultaneous 
execution of code is viewed as the concurrent expression of two genes, i.e., the chemical action of two proteins. The first new instruction allows a program to initiate a new expression: fork-th. Its execution creates a new instruction pointer ("forking off a thread") which immediately executes the next instruction, while the original thread skips it. Thus, fork-th is the rough equivalent of a promoter sequence in biochemistry. In a sense, this secondary expression is rather trivial and leads to redundancy; if the second thread is not sufficiently altered by the instruction following the fork-th, it simply executes the identical code as the first thread in lock-step. Of course, we are interested in how the organisms use this redundancy as a starting point to diversify the expression.

The second new instruction inhibits an expression: kill-th removes the instruction pointer which executed it, while the third addition id-th identifies which pointer is currently executing the code, i.e., which pattern is currently being expressed. We expect the three commands together to be useful in the regulation of expression. In principle, more than two instruction pointers can be generated by repeated issuing of the fork-th command, but here we restrict ourselves to a maximum of two threads in order not to complicate the analysis. In nature, of course, complex genomes express hundreds of proteins simultaneously.

As our experiments begin with a self-replicating program which does not use any of the multiple expression commands, the first question might be whether or not multiple expression will develop at all. In fact, it does almost instantly, as secondary expression (typically in the trivial mode mentioned earlier) appears to be immediately beneficial, perhaps in the same manner as simple gene doubling or a second promoter sequence. From here on, differentiation evolves, i.e., the two instruction pointers begin to adapt independently, to express more and more different code. Ultimately one might expect that each pointer executes an entirely different section of code, achieving local separation of genes and fully parallelized execution. The mode and manner in which this separation occurs is the subject of this investigation.

Several hundred independent experimental trials and controls were obtained in this study, testing different experimental conditions. For each of these trials we keep a record of a variety of statistics, including the dominant genotype at each time step, from which we can track the progression of evolution of the population, in particular by studying the details of its expression patterns.

\subsection{Basic Analysis Metrics}

In order to track the differentiation of the threads, we need to develop a means to monitor the divergence between the two instruction pointers roaming the genome. Also, to study the evolutionary pressures such as the mu- 
tational load, we need to introduce some standard (and some less standard) observables which allow us to track the adaptability of the population. This is one of the major advantages of digital chemistries - some of the data that we collect is impossible to accurately obtain in biochemical systems, and even less practical to analyze.

Fitness is measured as the number of offspring a genome produces per unit time, normalized to the replication rate of the ancestor. Thus, in all experiments the fitness of the dominant genotype starts at one and increases. Fitness improvements are due to two effects: the optimization of the gene for replication (the "copy-loop") leading to a smaller gestation time, as well as the development of new genes which accomplish computations on externally provided random numbers. These computations are viewed as the equivalent of exothermic catalytic reactions mediated by the expression products. We reward the accomplishment of all bit-wise logical operations performed on up to three numbers by speeding-up the successful organism's CPU at a rate commensurate to the difficulty of the computation.

Fidelity is the probability for an organism to produce an offspring perfectly identical to itself, i.e., the probability that the offspring is unaffected by mutations during the copy process. For pure copy-mutations (each instruction copied is mutated with a probability $R_{c}$ )

$$
F=\left(1-R_{c}\right)^{\ell}
$$

where $\ell$ is the organism's sequence length. In an adapting population, other factors can affect the fidelity and lead to low-fidelity organisms even while the theoretical fidelity is high. On the other hand, the development of errorcorrection schemes could increase the actual fidelity.

Neutrality $\nu$ is the probability that an organism's fitness is unaffected by a single point mutation in its genome. This is calculated by obtaining all possible one-point mutations of the examined genome, and processing each of them in isolation to determine fitness. The neutrality is then the number of neutral mutations divided by the total tested:

$$
\nu=\frac{N_{\text {neut }}}{\ell(\boldsymbol{D}-1)},
$$

where $\boldsymbol{D}$ is the number of different instructions in the digital chemistry, i.e., the size of the instruction set.

The preceding three indicators are key in determining the ability of an organism to thrive in an avida environment. Fitness, fidelity, and neutrality correspond respectively to an organism's ability to create offspring, for those offspring to have a minimum mutational load, and for them to survive those mutations which they do bear. Apart from this, however, there is another aspect which is necessary for a phylogenetic branch to be successful, and that is its ability to further adapt to its environment. To characterize this, we define two more genomic attributes: 
Neutral Fidelity is a measure which can be calculated once an organism's neutrality is known. It is the probability that an organism will give birth to an identical or equivalent offspring. Taking $f_{c}=R_{c}(1-\nu)$ to be the probability for a line to be mutated and be non-neutral to the organism, we obtain the neutral fidelity as:

$$
F_{\text {neut }}=\left(1-f_{c}\right)^{\ell} .
$$

Genomic Diffusion Rate is the probability for an offspring to have a genome different from its parent, but to be otherwise equivalent (i.e., neutral.) This is obtained by subtracting the genome's fidelity from its neutral fidelity

$$
D_{g}=F_{\text {neut }}-F .
$$

This is a particularly important indicator as it is the rate at which new, viable genotypes are being created, which in turn is the pace at which genetic space is being explored, and therefore directly proportional to the rate of adaptation.

\subsection{Differentiation Measures}

The following measures and indicators keep track of code-differentiation. In biochemistry, the differentiation of expression can be very varied, and includes overlapping reading frames (in-phase and out-of phase), overlapping operons and promoter sequences, and gene regulation. Obviously, there are no reading frames in our digital chemistry, but it is possible for a sequence of instructions to give rise to a different computation depending on which thread is executing it, in particular if one gene contains another (as is very common in overlapping biochemical genes [16]). Also, thread-identification may lead one thread to execute instructions which are skipped by the other thread, and threads may interact to turn each other on and off-a case of digital gene regulation. All such differentiation however has to evolve from the trivial secondary expression discussed earlier, and we consequently need to monitor the divergence of thread-execution with suitable measures.

Expression Distance is a metric we use to determine the divergence of the two instruction pointers. Simply put, this measurement is the average distance (in units of instructions) between the sections of the genome actively being expressed by the individual threads. At the initial point leading to secondary expression, this distance is zero as the two threads execute the same code in lock-step. If this value is high relative to the length of the genome, it is a strong indication that the instruction pointers are expressing different sections of the genetic code at any one time, while if it is low, they most likely move together with identical (or nearly so) execution patterns. However, this measure only indicates the differentiation between execution at 
a particular point in time, implying that if the execution is simply time-offset, this metric may be misleading.

Expression Differentiation distinguishes execution patterns with characteristically differing behavior. Each execution thread is recorded with time, and a count is kept of how often each portion of the genome is expressed. The expression differentiation is the fraction of the genome in which those counts differ. Thus, the ordering of execution (time-delay) is irrelevant for this metric; only whether the code ends up getting expressed differently by one thread vs. the other is important.

\subsection{Information Theoretic Measures}

We use information theory in order to distinguish sequences which do or do not code for genes. In our digital chemistry, regions which do not code for a gene are either unexecuted, i.e., the instruction pointer skips over them, or else neutral implying that their execution will typically not affect the behavior of the program. Trivial neutral instructions often involve the nop instructions (see Table I) which perform no function on their own when executed, but do act to modify other instructions. Thus, even though their execution is neutral their particular value can still severely affect the functioning of the organism. A perfectly neutral position sports any of the $\boldsymbol{D}$ instructions with equal probability among a population of sequences, while a maximally fixed position can only have one of the $\boldsymbol{D}$ instructions there. To distinguish these, we define the

Per-Site Entropy of a locus by trying out each of the $\boldsymbol{D}$ instructions at that position and evaluating the fitness of the resulting organisms. All neutral positions are assigned an equal probability to be expected at that site, while deleterious mutations are assigned a vanishing probability (as they would be selected against). Due to the uniform assignment of probabilities, the per-site entropy of locus $x_{i}$ (normalized to the maximum entropy $\log (\boldsymbol{D})$ ) is

$$
H\left(x_{i}\right)=\frac{\log N_{\text {neut }}\left(x_{i}\right)}{\log (\boldsymbol{D})}
$$

In an equilibrated population, this theoretical value of the per-site entropy is a good indicator for the actual per-site entropy, measured across the population (if the population is large enough). As positive mutations are extremely rare and we are only interested in the diversity of the population when it is in equilibrium, for the purposes of this measurement they are treated as if they were neutral. An indicator for the randomness within a sequence is the 
Per-Genome entropy, which we approximate by the sum of the per-site entropies

$$
H=\sum_{i}^{\ell} H\left(x_{i}\right)
$$

The actual per-genome entropy is in fact smaller, as the above expression neglects epistatic effects which lead to correlations between sites. For most purposes, however, the sum of the per-site entropies is a good approximation for the randomness. Measuring the entropy of the population by recording the individual genomic abundances is fruitless as the sampling error is of the order of the entropy [4].

\section{Single Expression vs. Multiple Expression}

Let us first examine adaptability as measured by the average increase in fitness for both single and multiple expression chemistries. In Fig. 11 A, the fitness is averaged for the 200 trials $\$$ which were seeded with small $(\ell=20)$ seed sequences and no size constraint (set I), for each of the chemistries. While the average increases relatively smoothly in time 2 , it should be noted that each individual fitness history is marked by periods of stasis interrupted by sharp jumps, giving rise to a "staircase" picture reminiscent of the adaptation of E. coli [5]. During adaptation, the sequence length increases commensurately with the acquired information, as shown in Fig. $1 \mathrm{~B}$.

Clearly, the trials in which multiple expression is possible adapt more slowly than the single-expression controls, a behavior that may appear at first glance to be paradoxical as the only difference in the underlying coding of the multiple expression trials is an increased functionality. However, as we have noted previously, the neutral fidelity of an organism directly determines the fraction of its offspring which are viable. As this value is inversely correlated to the length of the genome, there is a pressure for the genomes to evolve towards shorter length. Normally, this pressure is counteracted by the adaptive forces which require the organism to store more information in its genome, requiring increased length. Overlapping expression patterns (here, multiple parallelized execution) allows this adaptation to occur while minimizing the length requirement. Hence, multiple-expression genomes adapt more slowly.

\footnotetext{
${ }^{1}$ Each trial is seeded with a single ancestor, which quickly multiplies to reach the maximum number of programs in the population, set to 3,600 for these trials. The population was subjected to copy mutations at a rate of $7.5 \times 10^{-3}$ per instruction copied, and a rate of $0.5 \%$ of single insert or delete mutations per gestation period.

2 Time is measured in arbitrary units called updates. Every update represents the execution of an average of 30 instructions per program in the population.
} 

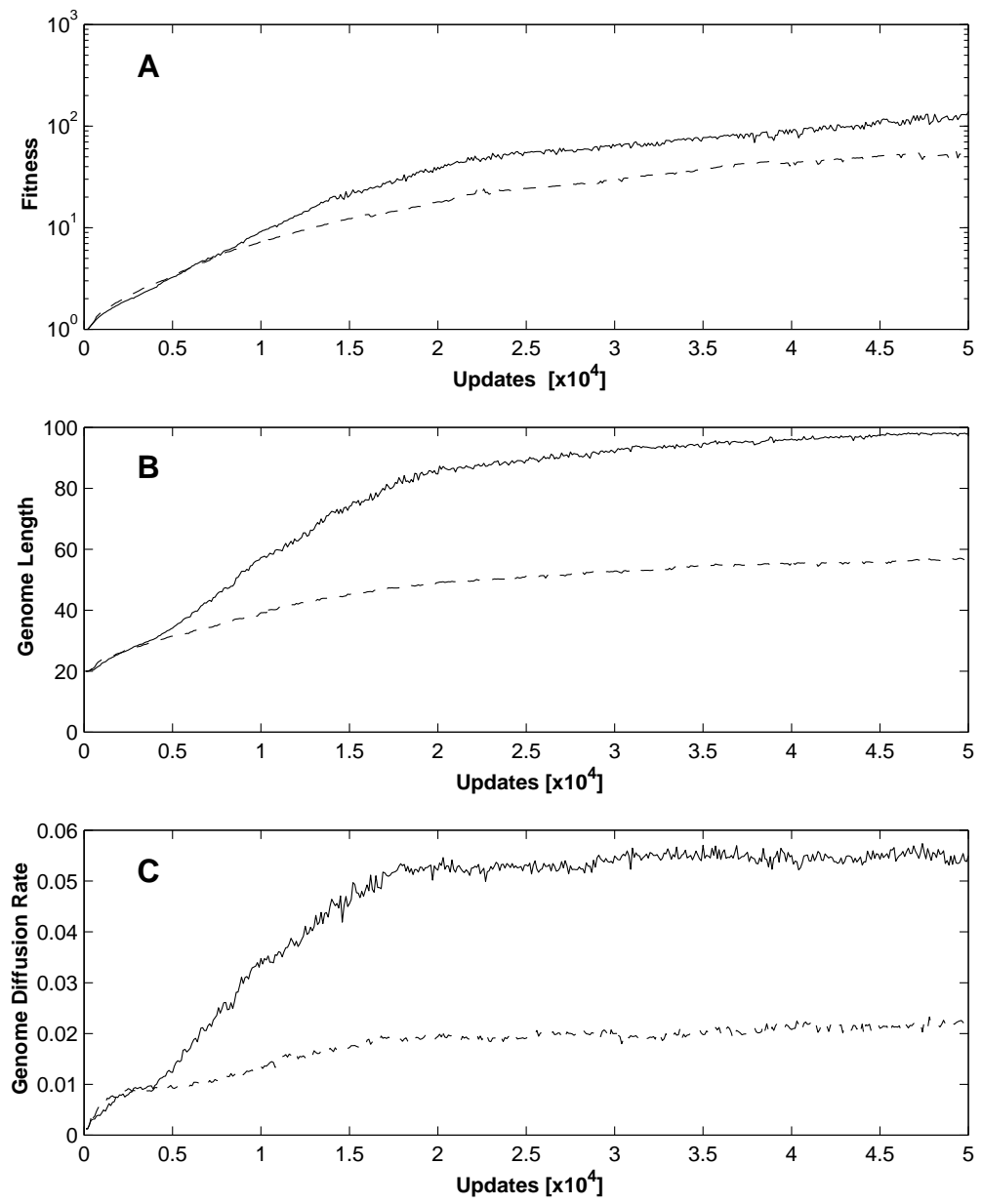

Fig. 1. (A): Average fitness as a function of time (in updates) for 200 populations evolved from $\ell=20$ ancestors, their average sequence length (B) and the average genomic diffusion rate $(\mathrm{C})$ for the single expression chemistry controls (solid line) and the multiple expression chemistry (dashed line).

The pitfalls of compacting so much information into the same portion of the genome are illustrated in Fig. 1 $1 \mathrm{C}$ where we plot the average genomic diffusion rate $D_{g}$ for both chemistries. It is evident in this graph that initially both sets of experiments explore genetic space at a comparable rate, but around approximately 5000 updates (on average) the diffusion rates diverge markedly, followed by a corresponding divergence in the fitness of the organisms (that a higher diffusion rate leads directly to higher fitness in an information-rich environment is shown in [3].) Investigating the course of evo- 
lution further, we see that it is precisely at this point that the differentiated, yet overlapping, use of multiple threads is typically established.

To further implicate overlapping expression in reduced adaptation for the populations, we consider (as was done in Ref. [7] for the bacteriophage $\Phi X 174)$ ) the substitution rate of instructions for overlapping versus nonoverlapping genes. The substitution rate in avida is equal to the neutrality (at equilibrium). We find the substitution suppression (the neutrality in multiply expressed code divided by the neutrality in singly expressed code) to be between 0.53 and 0.56 for the three sets of trials (Table II), similar (but not quite as severe) as the suppression ratio of between 0.4 and 0.5 observed in the bacteriophages [7]. This was to be expected, as there are no reading frames in avida which implies that two non-differentiated threads do not constrain the evolution any more than a single thread. When the instruction pointers do adapt independently and the threads differentiate, neutrality is compromised. Consequently, the instructions within sections of overlapping code are comparatively "frozen" into their state.

Table 2. Average neutrality of the final dominant genotype: multiply-expressed code (column 1), singly expressed code (column 2), and their ratio (column 3 ), for 200 populations grown from $\ell=20$ ancestors (variable length) [set I], 100 populations grown from $\ell=80$ ancestors (variable length) [set II], and 100 populations grown from $\ell=80$ ancestors (constant length) [set III].

\begin{tabular}{|c||c|c|c|}
\hline Set & $\nu_{\text {mult }}$ & $\nu_{\text {single }}$ & ratio \\
\hline I & 0.109 & 0.202 & 0.539 \\
II & 0.197 & 0.346 & 0.569 \\
III & 0.082 & 0.145 & 0.566 \\
\hline
\end{tabular}

\section{Evolution of Differentiation}

Let us now track the evolution of differentiation in more detail. We first address the de novo evolution of multiple expression, i.e., the development of multi-threading from linear execution. This question has previously been addressed within tierra 12], a population of self-replicating computer programs that served as the inspiration to our avida. In initial experiments, usage of multiple threads would not evolve spontaneously, but hand-written programs that had secondary expressions would evolve towards multiple expression 115$]$. More recently, experiments were carried out within a network version of the tierra architecture, which showed that a program which used different instruction pointers to execute different genes would not lose this ability [13]. The failure of multiple expression to evolve spontaneously in this system can be 
tracked back to problems with tierra's digital chemistry and the lack of an information-rich environment [11].

Within avida, the ability to use more than a single thread begins to develop within the first 5000 updates and is very common after about 10,000 updates, depending on the experimental boundary conditions. Fig. 2 A shows the (averaged) percentage of a program's lifetime in which more than one thread is active, for the populations of set I (solid line), set II (dashed line) and set III (dotted line). It is apparent that multiple expression develops much more readily in smaller genomes, due to the fact that the logistics are less daunting.

In panels $\mathrm{B}$ and $\mathrm{C}$ of Figure 2 we display two indicators of differentiation (defined earlier), the expression distance and the expression differentiation, respectively. The expression distance appears to be sensitive to the experimental starting condition, as set II and set III show a value over twice that of set I. We observe that this is due to the small size of the ancestor used in set I: as that ancestor develops threading very quickly, it loses adaptability earlier and lags both in average fitness and average sequence length. In fact, those averages are dragged down by a significant percentage of the trials in set I which were stuck in an evolutionary dead-end. Set II and III were seeded with an ancestor of length $\ell=80$ and did not suffer from this lot. Fig. 2 2 C shows the expression differentiation, i.e., the fraction of code that is executed differently by the two threads. This fraction is less dependent on experimental conditions, and the genomes appear to develop towards 0.5. Note, however, that this measure cannot accurately reflect differentiation which is more subtle than threads executing particular instructions a different number of times. For example, two threads which execute a stretch of code in an identical manner but that start execution at different points "upstream" may end up calculating very different functions, and thus have quite different behaviors. This difference will thus be underestimated. While the preceding graphs seem to indicate that differentiation stops about half-way through the duration we record, this is actually not so, as the more microscopic analysis of the following section reveals. Finally, Fig. 3 shows the evolution of the fraction of code that is executed by multiple threads.

We anticipate that this fraction rises swiftly at first, but then levels off, as it is not advantageous to multiply express all genes (see below). However, we might anticipate that the fraction would start to decline at some point, when the organism develops the ability to localize its genes and use independent instruction pointers for each of them. We do not witness this trend in Fig. 3 presumably because there is no cost associated with the development of secondary expression. This should be viewed as a peculiarity of the digital environment rather than a universal feature, which we hope to eliminate with future refinements of the avida world. 

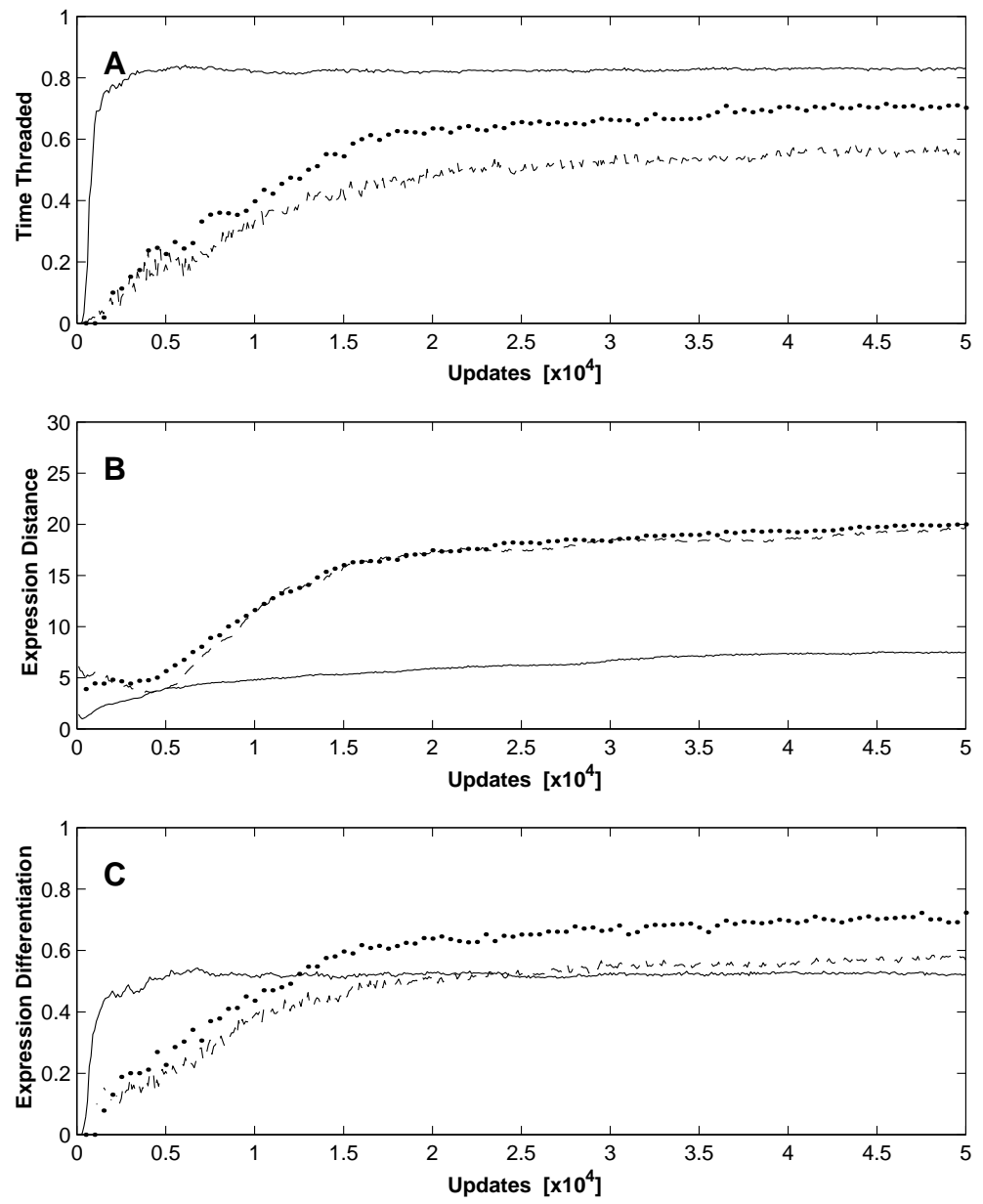

Fig. 2. Differentiation measures. (A): Average fraction of lifetime spent with secondary expression, as a function of time (in updates), (B): average expression distance, (C): average expression differentiation. Set I (solid line), set II (dashed line), and set III (dotted line).

\section{Evolution of Genetic Locality}

To get a better idea of how evolution is acting upon programs harboring multiple threads, we must look at exactly what is being expressed. We can loosely characterize all organisms by tracking three separate genes. They are "self-analysis" (slf), "replication" ( $r p l)$, and "computation" ( $\mathrm{cmp}$ ). To follow the progression of these genes through time, we examine a sample experiment seeded with an ancestor of size 80 (as before, capable only of self-replication), in an environment in which size-altering mutations are strictly forbidden (a 


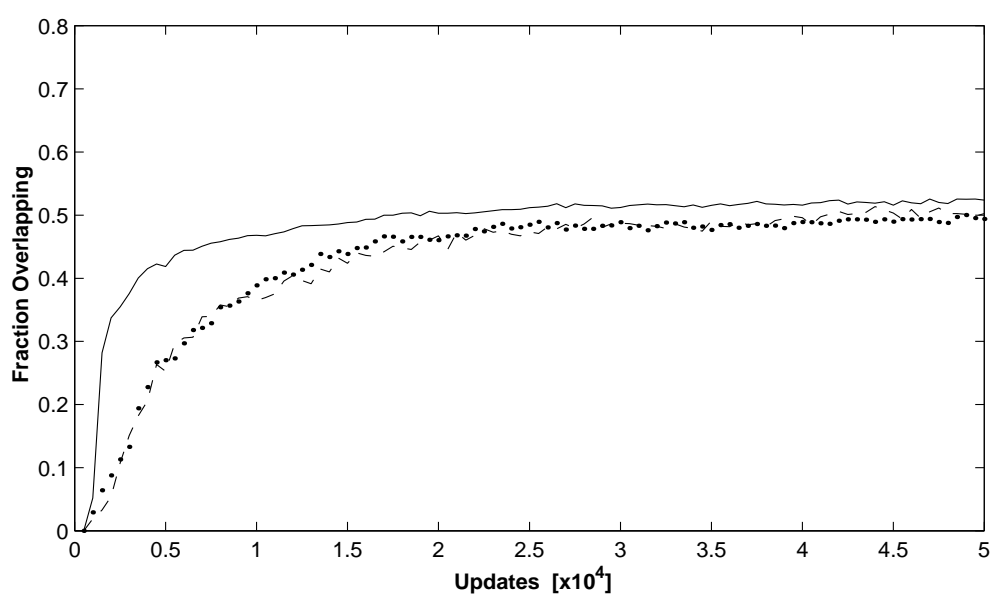

Fig. 3. Average fraction of doubly expressed code for the three experimental sets. Solid line: set I, dashed line: set II, dotted line: set III.

trial from set III). This limitation was enforced in order to better study the functionality of the organism and the location of its genes. Similar studies have been done with all 400 trials used to collect the bulk of the data for this report, showing comparable behavior.

In Fig. A A we follow the per-site entropies for each locus as a function of time. Positions are labeled by 1 to 80 on the vertical axis, while time proceeds horizontally. A grey-scale coding has been employed to denote the variability of each locus, where the white end denotes more variable positions and the dark end more fixed positions. Because the per-site entropies have been calculated by obtaining the frequency with which each instruction appears at that locus within the population (as opposed to the theoretical estimate based on neutrality), major evolutionary transitions are identifiable by dark vertical bands. Fig. AB shows which portion of the code is expressed by which pointer, by two pointers simultaneously, or not at all.

The first gene slf uses pattern matching on nop instructions in order to find the limits of its genome and from that calculate it's length. This value is used for elongation (via the command alloc), which adds empty memory to the genome and prepares it for the "execution" of the replication gene. Note that avidian genomes are circular. There are two interesting points to note about the evolution of slf: First, there are many methods by which the organism can determine its own genomic length, so this gene tends to vary widely. Most of the time the organism keeps pattern matching techniques, but matches different portions of the code. However, often an organism shifts to purely numerical methods performing mathematical operations upon itself which yield the genome length "by accident". The other evolutionary characteristic of this gene is that there is no benefit in expressing it multiple times as it 

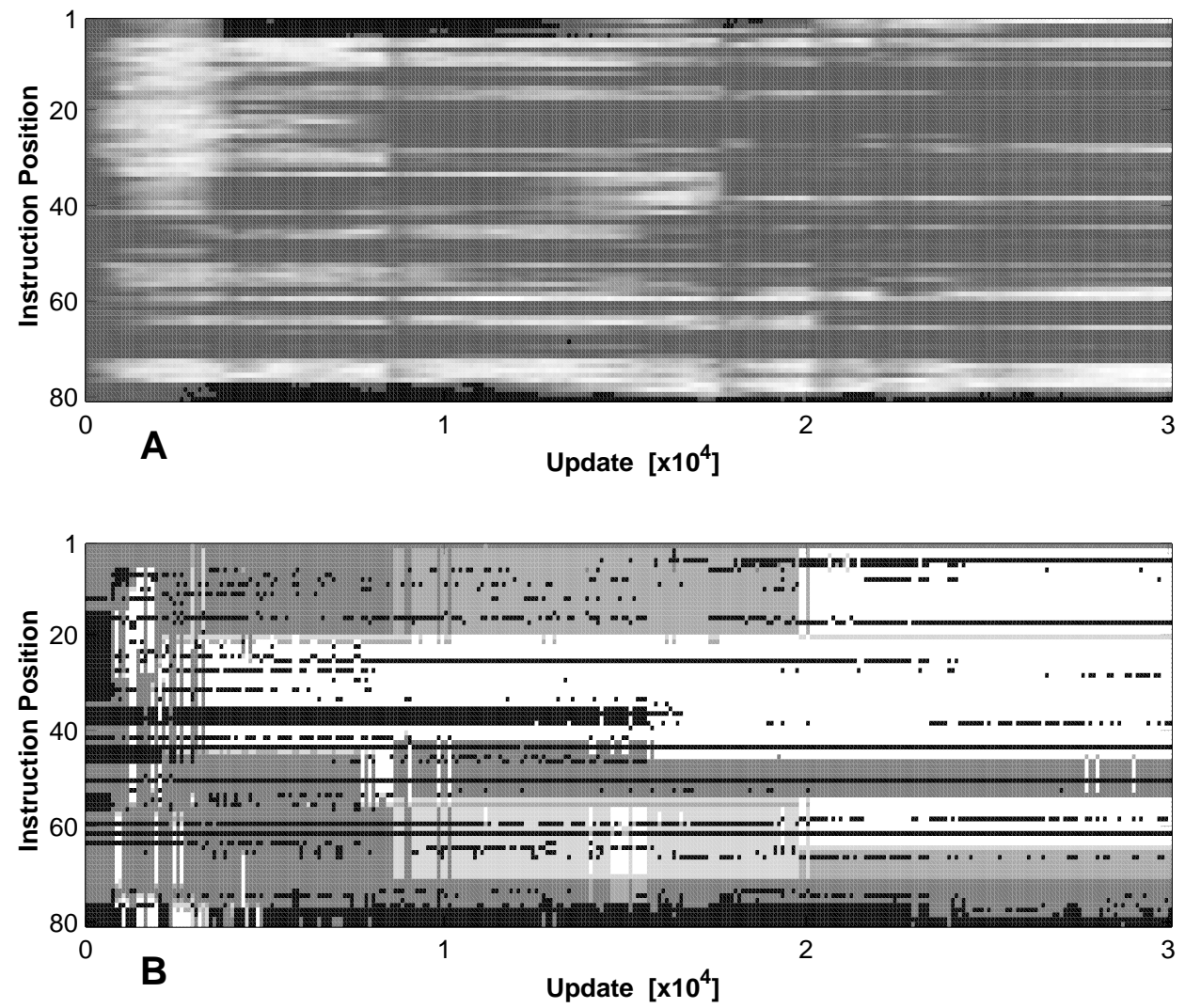

Fig. 4. (A): Per-site entropy for each locus as a function of time for a standard (set III) trial. Random (variable) positions with near-unit per-site entropy are bright, while "fixed" instruction with per-site entropy near zero are dark. (B): Thread identification within a genome. Black indicates instructions which are never directly executed, dark grey denotes instructions executed by a single thread when no other thread is active, while sections which are executed by a single thread while another thread is executing a different section are colored in lighter shades of grey. Sections with overlapping expression are in white.

has a fixed result which needs only be applied once during the gestation cycle. Looking at Figure 1 , the slf gene initially spans from lines 44 to 61 plus the first four lines and last four lines of the genome which are boundary markers fashioned from nop instructions. The first major modification to the slf gene occurs around update 3000 . The pattern used to mark the limits of the genome is a series of four nop-A instructions. As a newly allocated genome has all of its sites initialized to nop-A, the genome is re-organized such that these lines are no longer copied. This reduces the possibility of variation in 
these sections of code to zero. This is apparent in Fig. AA as the positions of these limit patterns become completely black indicating vanishing entropy.

The slf gene is continuously undergoing minor changes as is becomes more optimized to require fewer lines of code to perform its function. Near update 13,000 it shifts dramatically and is replaced by one in which size is calculated using only the final boundary markers. The distance from the gene to the final marker is determined, and then manipulated numerically in order obtain the number which is the size of the organism. Looking at the first four lines of Fig. 1 A around this update, we see that they are slowly phased out and increase in entropy as they are no longer as critical to the organism's survival. Finally the size of the pattern marking the end boundary of the organism is shortened until it becomes only a single line. By the end of the evolution shown, the slf gene only occupies lines 48 through 56 . Note that all of these lines are only expressed a single time.

The next gene under consideration is the actual replication gene $r p l$. This sequence of instructions uses the blank memory allocated in the self-analysis phase and enters a 'copy-loop' which moves line by line through the genome, copying each instruction into the newly available space. When this process is finished, it severs the newly created copy of itself which is then placed in an adjacent lattice site. These dynamics spawn off a new organism which, if the copy process was free of mutations, would be identical to the parent. In Fig. 1, the organism being tracked has its replication gene on lines 65 to 71 until update 24,000 at which time this gene actually grows an additional line becoming much more efficient by "unrolling" its copy-loop. What this means is that it is now able to copy two lines each time through the loop. From the dark color of these lines, it is obvious that they have very low entropy, and are therefore very difficult to mutate. The copy-loop is a very fragile portion of code, critical to the self-replication of the organism, yet we do see some evolution occurring here when multiple threads are in use. Often the secondary thread will simply "fall through" the copy-loop (not actually looping through to copy the genome) and move on to the next gene, while the other thread performs the replication. However, sometimes the two threads will actually evolve together to use the copy loop in different ways, with each thread copying part of the genome. In Fig. 1 , most of the rpl gene is executed by only one thread. The $r p l$ gene is followed by junk code which, while executed sporadically, does not affect the fitness in any way (as evidenced by the light shading in Fig. (1) for these lines).

The most interesting of the genes is the computation gene cmp. The ancestor does not possess this gene at all, so it evolves spontaneously during the adaptive process. There are 78 different computations rewarded in this environment, all of which are based on bit-wise logical operations. The organisms have three main commands which they use to accomplish those: a get instruction which retrieves numbers from the environment, a put instruction to return the processed result, and a nand instruction which computes the log- 
ical operation not-and (see Table I). Any logical operation can be computed with a properly arranged collection of nand instructions.

The $c m p$ gene(s) evolve uniquely in each trial, enabling the organisms to perform differing sets of tasks. There are, however, certain themes which we see used repeatedly whereby the same section of code is used by both threads, but their initial values (i.e., the processing performed thus far on the inputs) differs. Consequently, this section of code performs radically different tasks, actually encouraging this overlapping. Portions of this algorithm which might have some neutrality for a single thread of execution will now be frozen due to the added constraints imposed by a secondary execution. The size of $c m p$ grows during adaptation as a number of computations are performed, and the gene is almost always expressed by both threads as this is always advantageous. In Fig. A, the $c m p$ gene stretches from line 1 to line 42 (at update 30,000 ), while it is considerably smaller earlier. Furthermore, the genome manages to execute the entire gene by both threads (the transition from single expression of part of $\mathrm{cmp}$ to double expression is visible around update 20,000). This gene ends up being expressed many times (as the instruction pointers return to this section many times during execution). All in all, 17 different logical operations are being performed by this gene.

By the end of the evolution tracked in Fig. 1, most of the genes appear to occupy localized positions on the genome. The $\mathrm{cmp}$ gene (white sections in Fig. (1) is revisited many times by both threads with differing initial conditions for the registers, allowing the genome to maximize the computational output. In the meantime, those sections have become fixed (their variability is strongly reduced) as witnessed by their dark shading in Fig. (1) A.

\section{Discussion and Conclusions}

The path taken by evolution from simple organisms with few genes towards the expression of multiple genes via overlapping and interacting gene products in complex organisms is difficult to retrace in biochemistry. Artificial Life, the creation of living systems based on a different chemistry but using the same universal principles at work in biochemical life, may help to understand some key principles in the development of gene regulation and the organization of the genetic code. We have examined the emergence and differentiation of code expression in parallel within a digital chemistry, and found some of the same constraints affecting multiply expressed code as those observed in the overlapping genes of simple biochemical organisms. For example, multiply expressed code is more fragile with respect to mutations than code that is "transcribed" by only one instruction pointer, and as a result evolves more slowly. During most stages of evolution, two constraints are most notable: the pressure to reduce sequence length in order to lessen the mutational load, and the pressure to increase sequence length in order to be able to store more information. Simple organisms can give in to both 
pressures by using overlapping genes, gaining in the short term but mortgaging the future: the reduced evolvability condemns such organisms to a slower pace of adaptation, and exposes them to the risk of extinction in periods of changing environmental conditions.

This trend is clearly visible in the evolution of digital organisms, as is a trend towards multiple expression of as much of the code as possible. This latter feature we believe not to be universal, but rather due to the fact that multiple expression in avida is cheap, i.e., no resources are being used in order to express more code. In a more realistic chemistry, this would not be the case: adding an instruction pointer should put some strain on the organism and use up energy; in such circumstances multiple expression would only emerge if the advantage of the secondary expression outweighs the cost of it. We also expect more complex gene regulation in such an environment, as genes would be turned on only when needed.

Still, under extreme conditions we believe that multiple overlapping genes are a standard path that any chemistry might follow. Even though evolution slows down, such organisms can be rescued either by the development of error-correction algorithms, or an external change in the error rate. In either case, a drastic reduction of the mutational load would enable the sequence length to grow and the overlapping genes to be "laid out" (for example by gene-duplication). The corresponding easing of the coding constraints might give rise to an explosion of diversity and possibly the emergence of multicellularity.

Acknowledgements. We would like to thank Grace Hsu and Travis Collier for collaboration in the initial stages of this work. Access to a Beowulf system was provided by the Center for Advanced Computing Research at the California Institute of Technology. This work was supported by the National Science Foundation.

\section{References}

1. Adami, C. (1995) Learning and complexity in genetic auto-adaptive systems, Physica D 80, 154.

2. Adami, C. (1998) Introduction to Artificial Life, Telos Springer Verlag, New York.

3. Adami, C., Collier, T. C. and Ofria, C. (1999) Robustness and evolvability of computer languages, to be published.

4. Basharin, G. P. (1959) On a statistical estimate of the entropy of a sequence of independent random variables, Theory Probability Appl. 4, 333.

5. Elena, S. F., Cooper, V. S., and Lenski, R.E. (1996) Punctuated evolution caused by selection of rare beneficial mutations, Science 272, 1802.

6. Keese, P. and Gibbs, A. (1992) Origins of genes: "Big bang" or continuous creation?, Proc. Natl. Acad. Sci. 89, 9489-9493.

7. Miyata, T., and Yasunaga, T. (1978) Evolution of overlapping genes, Nature $\mathbf{2 7 2}, 532$. 
8. Mizokami, M., Orito, E., Ohba, K., Lau, J. Y. N., and Gojobori, T. (1997) Constrained evolution with respect to gene overlap of Hepatitis B virus, J. Mol. Evol. 44(Suppl. 1), S83-S90.

9. Normark, S., Bergström, S., Edlund, T., Grundström, T., Jaurin, B., Lindberg, F. P., and Olsson, O. (1983) Overlapping genes, Ann. Rev. Gen. 17, 499-525.

10. Ofria, C., Brown, C. T., and Adami, C. (1998) Avida User's Manual, in [2].

11. Ofria, C., Collier, T. C., Hsu, G., and Adami, C. (1999) Evolution of differentiated expression patterns in digital organisms, KRL preprint MAP-250 (February 1999).

12. Ray, T. S. (1992) An approach to the synthesis of life, in Proc. of Artificial Life II, C. G. Langton, C. Taylor, J. D. Farmer, and S. Rasmussen, Eds., Addison Wesley, Redwood City, p. 371.

13. Ray, T. S. and Hart, J. (1998) Evolution of differentiated multi-threaded digital organisms, in Proc. of Artificial Life VI, C. Adami, R. K. Belew, H. Kitano, and C. E. Taylor, Eds., MIT Press, Cambridge, MA, p. 295.

14. Samuel, C. E. (1989) Polycistronic animal virus messenger RNAs, Prog. Nucleic Acids Res. Mol. Biol. 37, 127-153.

15. Thearling, K. and Ray, T. S. (1994) Evolving multi-cellular artificial life, in Proc. of Artificial Life IV, R. A. Brooks and P. Maes, Eds., MIT Press, Cambridge, MA, p. 283.

16. Watson, J. D., Hopkins, N. H., Roberts, J. W., Steitz, J. A., and Weiner, A. M. (1987) Molecular Biology of the Gene, Fourth Ed., Benjamin Cummings, Menlo Park, CA, p. 457. 